\title{
Mortalidade hospitalar como indicador de qualidade: uma revisão
}

\author{
Hospital mortality as an indicator \\ of clinical performance: a review
}

Claudia Travassos 1

José Carvalho de Noronha 2

Mônica Martins 3

${ }^{1}$ Departamento de Informações em Saúde, Centro de Informação Científica e Tecnológica, Fundação Oswaldo Cruz, Av.Brasil 4365,

Manguinhos, 21045-900 Rio de Janeiro, RJ, Brasil claudia@malaria.procc.

fiocruz.br

2 Instituto de Medicina Social, Universidade Estadual do Rio de Janeiro, Centro de Informação Científica e Tecnológica, Fundação Oswaldo Cruz

3 Departamento de Administração e

Planejamento em Saúde, Escola Nacional de Saúde Pública, Fundação

Oswaldo Cruz
Abstract This article discusses the principal methodological problems related to hospital mortality as an indicator of clinical performance. Hospital mortality rates variation are due to various factors associated with patients characteristics and to the specific diseases they are suffering. Socio-demographic variables, presence of comorbidity and severity may define case-mixes were chances of dying are not associated to technology deployed or quality of care. Relevant methodological aspects for calculating the rates include the quality of the source of data, time period and aggregation criteria. Various models that exist both for classifying severity of cases and for risk adjustment are presented and discussed. Explanatory models for mortality rates variation are also examined. The authors conclude that outcome indicators can be used as tool for health care service evaluation. For those conditions which death is not a rare event hospital mortality rates may constitute an useful tool for indicating services with low than expected quality of care.

Key words Quality; Health Services Evaluation; Outcome Indicators; Hospital Mortality; Rate Adjustment
Resumo Este artigo visa a discutir as principais questões metodológicas relacionadas à mortalidade hospitalar como indicador de qualidade. Variações nos valores deste indicador se devem a inúmeros fatores, associados ao doente $e$ à doença, que devem ser examinados para que possamos utilizá-lo como medida de desempenho. Presença de comorbidades e a gravidade do caso estão associadas à chance de morrer. Aspectos metodológicos, relevantes para a construção deste indicador, incluem a qualidade das fontes de dados, o intervalo de tempo no qual elas são calculadas e os diferentes tipos de agregação. São discutidos diversos modelos, tanto para classificação da gravidade, quanto para o ajuste das taxas de mortalidade entre serviços. São examinados ainda modelos explicativos para a variação de mortalidade. Conclui-se que nas condições em que a morte não é um evento raro, o emprego de taxas de mortalidade hospitalar representa uma ferramenta útil para indicar serviços com eventuais problemas de qualidade.

Palavras-chave Qualidade; Avaliação de Serviços de Saúde; Indicadores de Desempenho; Mortalidade Hospitalar; Ajuste de Taxas 


\section{Introdução}

Nos últimos anos se intensificaram os processos de revisão da organização e do funcionamento de sistemas de saúde públicos e privados em diversas partes do mundo. $\mathrm{O}$ termo reforma setorial, usado com múltiplos sentidos, ganhou amplo trânsito. Em recente estudo sobre os projetos de reforma existentes, realizado por integrantes da Organização para Cooperação Econômica e Desenvolvimento (OECD), foram identificados três principais grupos de orientações sobre políticas de saúde: a busca da eqüidade e o aumento da eficiência, macro e microeconômica (OECD, 1995). Também foi constatado que a maior parte das iniciativas de reforma dos sistemas de saúde almejava controlar as despesas e aumentar a eficiência dos cuidados prestados (Anderson \& Erickson, 1987; Schieber \& Pollier, 1987; Saltman \& Otter, 1989; Hurst, 1991; WHO, 1993; Contandriopoulos, 1994). Muitas das medidas adotadas visavam a estimular a competição dentro dos sistemas de saúde, descentralizando e flexibilizando os métodos de gestão e, portanto, pretendendo garantir maior liberdade de escolha aos usuários (Saltman \& Otter, 1989). Quando crescem as pressões sobre a disponibilidade de recursos para políticas sociais, ou quando a busca pela eqüidade é o objetivo central das propostas de reforma, aumenta a importância da avaliação dos cuidados de saúde prestados (Almeida, 1996) principalmente quando muitas das propostas e iniciativas procuram transferir para o jogo de forças de mercado a definição das melhores alternativas alocativas.

Nas décadas de 60 e 70, as avaliações de serviço de saúde privilegiaram os estudos baseados na análise do processo de cuidado. Leon Wyzewianski (1988) sinaliza, nas publicações dos autores clássicos, a predominância de trabalhos voltados para a análise de estrutura e processo. Estudos mais recentes enfatizam a análise de resultados, que tem como fundamento a existência de uma relação entre a assistência prestada, já terminada ou ainda em andamento, com a medida de resultado utilizada para esta avaliação (Donabedian, 1985; Brook \& McGlynn, 1991).

Principalmente nos Estados Unidos, as avaliações de qualidade dos serviços de saúde que utilizam o enfoque de resultado ganharam destaque a partir da criação de uma legislação específica, que impõe a avaliação da assistência médica prestada a todos os beneficiários dos programas governamentais Medicare (programa de saúde destinado às pessoas com mais de 65 anos e doentes renais em fase terminal) e Medicaid (programa de saúde para a população abaixo da linha de pobreza) (Blumberg, 1986; Fottler, 1987; Chassin et al., 1989a; Hartz et al., 1989). A partir de 1986, o Health Care Financing Administration (HCFA), órgão do governo americano responsável pela administração do Medicare e pela parte federal do $M e$ dicaid, passou a divulgar as taxas de mortalidade dos hospitais que atendem a população beneficiária do programa Medicare. Essas taxas foram analisadas para identificar os hospitais com possíveis problemas na qualidade do cuidado prestado ao paciente. Orientação semelhante foi seguida por organismos estaduais, como o monitoramento da mortalidade por cirurgia coronariana implantado pelo estado de Nova Iorque (Cleves \& Golden, 1996; Localio, 1997; New York State Department of Health, 1997).

A primeira divulgação das taxas de mortalidade hospitalar sofreu diversas críticas, que questionaram desde a precisão da codificação dos diagnósticos e procedimentos até a validade do modelo usado para prever o risco de morte em cada hospital. Desde então, foram introduzidas mudanças metodológicas na análise dos óbitos hospitalares como, por exemplo, a desagregação das taxas de mortalidade hospitalar por grupos diagnósticos.

Em 1988, o Centre for Health Economics de Iorque, na Inglaterra, publicou as taxas de mortalidade dos hospitais ingleses (Kind apud Mant, 1995) e, em 1994, o Sistema Nacional de Saúde do Reino Unido (NHS) publicou as taxas de mortalidade hospitalar na Escócia (Dillner apud Mant, 1995). Em 1997, foi anunciado que o NHS passaria a divulgar diversos indicadores de desempenho dos seus hospitais (Mckee, 1997). Algumas instituições hospitalares alemãs também passaram a produzir relatórios detalhados sobre os óbitos ocorridos naqueles hospitais (Editorial, 1993).

Um outro exemplo da difusão dos estudos de resultado é o projeto ORIX, da tradicional agência americana de acreditação de serviços de saúde Joint Commision on Accreditation of Healthcare Organizations (JCAHO), que visa a acoplar o monitoramento de indicadores de desempenho ao processo de acreditação, baseado na conformidade com padrões. 
Apesar do crescente uso de medidas de resultados na avaliação da qualidade da assistência médica, essa abordagem ainda apresenta limitações metodológicas importantes. Brook et al. (1977) destacam a existência de uma situação paradoxal, onde o aumento das demandas para a criação de sistemas de monitoramento de desempenho, com base em indicadores de resultado, confronta-se com a escassez de critérios e padrões válidos e confiáveis. Faltam referências que sirvam como medidas e como parâmetros de comparação, fundamentais para a avaliação da qualidade dos resultados observados.

\section{Indicadores de desempenho}

Indicadores de desempenho (performance indicator; clinical indicator) são medidas indiretas da qualidade utilizadas como instrumento de monitoramento para salientar os processos, serviços ou profissionais que podem estar apresentando problemas e que necessitam de uma avaliação mais direta. São informações expressas através de um evento, uma taxa ou uma razão. Os indicadores de desempenho são utilizados mais freqüentemente em atividades de monitoramento externo, que assumem papel crescente como meio de dar visibilidade, aos usuários e às agências financiadores e reguladoras, do alcance das atividades de melhoria de qualidade desenvolvidos nos serviços de saúde (Sheldon, 1998).

Os indicadores de desempenho podem medir tanto o processo de cuidado ao paciente quanto seu resultado. Este resultado são as mudanças, favoráveis ou desfavoráveis, ocorridas no estado de saúde do paciente, decorrentes do processo de cuidado (Donabedian, 1980). Eles também medem as diferentes dimensões de qualidade, como a adequação técnica do cuidado, a eficiência, a segurança, a eqüidade, etc.

É importante destacar que a utilidade de um indicador de desempenho depende de uma série de atributos, particularmente, da sua validade. A validade - grau com o que o indicador alcança seus objetivos - é expressa pela capacidade de identificar situações onde possam ocorrer problemas na qualidade do cuidado prestado ao paciente. Cabe ainda destacar que bons indicadores são os que apresentam forte associação, estabelecida com base no conhecimento científico, entre o processo de cuidado e a medida de resultado utilizada (valida- de causal). Entretanto, limitações no conhecimento sobre a eficácia dos procedimentos diagnósticos e terapêuticos restringem a determinação da validade causal de diferentes indicadores de resultado (Brook \& McGlynn, 1991). Na prática, grande parte dos indicadores de desempenho possuem apenas validade aparente (face validity) - o sentido das relações que devem indicar fazem sentido para especialistas Chassen et al., (1989b).

Portanto, a determinação da validade de um indicador de desempenho é fundamental para o desenvolvimento destes indicadores. Imprecisões na validade de um indicador implicam em imprecisões na sua interpretação. O problema central no desenvolvimento de indicadores de desempenho não é desenhálos, mas sim saber, de fato, o que eles expressam (O’Leary, 1995). Estas dificuldades têm levado ao reconhecimento de que o principal papel deste tipo de informação é fornecer conhecimento (informação) para o desenvolvimento das atividades que melhorem os processos de atendimento. No entanto, para vários indicadores, sua validade como instrumento de avaliação da qualidade dos serviços ainda é limitada (Berwick, 1996; Iezzoni, 1997; Sheldon, 1998).

\section{Mortalidade como indicador de desempenho}

A mortalidade hospitalar é um indicador de desempenho tradicional, expresso por uma taxa. A utilização de dados de mortalidade como indicador de qualidade em hospitais foi proposta há muitas décadas por Ernest Codman (Codman, 1916 apud Normand et al., 1996), um dos precursores na busca de instrumentos para a melhoria da qualidade dos serviços de saúde. Da mesma forma, no século passado, Florence Nightingale já propunha a coleta sistemática de dados sobre os pacientes internados como método para acompanhar e explicar as variações nas taxas de mortalidade entre hospitais (Nightingale, 1863 apud Fink et al., 1989). Mais recentemente, a utilidade da mortalidade hospitalar como indicador da qualidade da assistência foi destacada por Dubois et al., (1987a).

A morte hospitalar considerada evitável pode estar relacionada a diversas causas que incluem as infecções hospitalares, o uso inadequado de medicamentos e de outras tecnolo- 
gias, as falhas na supervisão, os erros durante a cirurgia ou as altas inapropriadas (OTA, 1988). Segundo Kahn e colaboradores (1988), dados sobre a mortalidade de pacientes durante ou logo após sua hospitalização podem atender a pelo menos dois propósitos: determinar se o desempenho de um hospital tem melhorado (ou deteriorado) ao longo do tempo, e monitorar se o desempenho de um conjunto de hospitais difere num dado período temporal.

$\mathrm{Na}$ avaliação das mortes hospitalares a preocupação central deve estar voltada para a identificação dos óbitos que poderiam ser evitados, uma vez que todo paciente apresenta um risco de morrer, que define as suas chances de sobrevida. Contudo problemas de qualidade no processo de cuidado ao paciente podem aumentar esse risco (Rosen, 1987; Dubois \& Brook, 1988).

Causas das variações - Diferenças nas taxas de mortalidade entre hospitais ou médicos podem ser atribuídas aos seguintes fatores (Iezzoni, 1994): diferenças quanto à gravidade do estado de saúde da população atendida em cada hospital; variações na eficácia das tecnologias médicas empregadas; adequação do processo de cuidado ao paciente; e erros aleatórios.

O problema metodológico central para explicar as variações observadas nas taxas de mortalidade hospitalar é identificar a importância relativa de cada um dos fatores acima. É por isso que a mortalidade hospitalar pode apresentar dificuldades de interpretação. $\mathrm{O}$ caminho para construir um modelo explicativo das variações nas taxas de mortalidade hospitalar entre serviços passa pela incorporação de variáveis que definam a população de pacientes e o perfil de gravidade dos casos internados, de forma que as diferenças na qualidade (eficácia das tecnologias e adequação do processo) representem apenas a variação remanescente.

Validade - A discussão sobre a validade da mortalidade hospitalar como medida da qualidade do cuidado se apoia em dois eixos principais: 1) a mensuração precisa e adequada da gravidade dos casos tratados e 2) o estabelecimento, com base em evidências científicas, das relações de causalidade entre o resultado (óbito) e o processo de cuidado prestado, isto é, o estabelecimento da validade causal (Donabedian, 1985).

A gravidade dos casos exerce forte influência sobre o resultado do cuidado prestado, e sua mensuração é um tema freqüente no debate sobre a validade das taxas de mortalidade hospitalar como forma de avaliação da qualidade. Posteriormente ela será discutida em mais detalhes.

Outro aspecto sobre a validade da mortalidade hospitalar como indicador de qualidade Kahn et al., (1988), relaciona-se ao fato de que a morte só é uma medida apropriada de qualidade do cuidado prestado em certas condições mórbidas, ou em determinados procedimentos. Como apontado anteriormente, qualquer medida de resultado representa uma forma indireta de avaliar o processo de cuidado e, portanto, só é válida se comprovadamente associada a este processo. Para pacientes com doenças tratáveis (como insuficiência cardíaca, infarto agudo do miocárdio ou pneumonias), uma taxa de mortalidade baixa pode refletir um bom atendimento. Ao contrário, para doentes com doenças terminais (por exemplo, neoplasias em estágio terminal), a morte pode ser um resultado inevitável. Portanto as taxas de mortalidade não refletem a qualidade da assistência. Para este grupo de doenças, o estado funcional do paciente e sua qualidade de vida podem ser medidas bem mais apropriadas para avaliar a qualidade do cuidado.

Além dos pontos destacados acima, devem ser consideradas outras questões sobre a validade da mortalidade hospitalar como medida da qualidade do cuidado (Dubois, 1987b; OTA, 1988; Roos et al., 1988; Fink et al., 1989; DesHarnais, 1990a; Flood, 1990; Kelly, 1990; Lohr, 1990; Luft et al., 1990; Krakaver et al., 1992, Romano, 1993, Iezzoni, 1994). São elas: a) a definição do espaço de tempo apropriado para estudar os óbitos hospitalares, ou seja, em que intervalo temporal o óbito pode ser atribuído à assistência hospitalar recebida; b) o nível de agregação do indicador, ou seja, se este deve ser calculado para todos os pacientes ou desagregado segundo o diagnóstico, grupo de diagnósticos ou procedimentos médicos ou cirúrgicos específicos; c) a validade do indicador decorrente da qualidade dos dados empregados, tanto no numerador e denominador, quanto daqueles usados como co-variáveis em modelos para predição.

Há questões teóricas e práticas envolvidas na definição do intervalo de tempo mais apropriado para o cálculo das taxas de mortalidade hospitalar, mas não existe consenso entre os autores. Alguns preferem contabilizar todos os óbitos ocorridos dentro de um inter- 
valo de tempo fixado na data de admissão (que poderia incluir óbitos ocorridos após a alta do paciente), outros trabalhar com uma medida mais tradicional, ou seja, apenas com a contabilização dos óbitos ocorridos durante a internação. Variações no tempo médio de permanência entre hospitais, diferenças nas práticas de admissão e de alta, acesso diferenciado ao cuidado domiciliar, a serviços de saúde para pacientes terminais e a hospitais psiquiátricos, podem influenciar o local de ocorrência do óbito (OTA, 1988). Estimuladas por uma prática gerencial voltada para maior eficiência, pelas forças de mercado ou pelos sistemas de reembolso da assistência, algumas instituições podem reduzir a permanência hospitalar, incentivando a alta precoce ou transferindo pacientes mais graves ou em estágios terminais. Os estabelecimentos hospitalares que realizam essas transferências, provavelmente, terão menores taxas de mortalidade intra-hospitalar. Mas fixar o intervalo de tempo no cálculo das taxas de mortalidade também apresenta riscos, como a inclusão nas taxas de óbitos de eventos não relacionados à qualidade do cuidado de saúde dos hospitais. Além disso, coletar informações referentes aos períodos de internação e pós-alta imediata, impõe o uso de outras fontes de dados além daquelas coletadas rotineiramente pelos hospitais, como, por exemplo, os dados das declarações de óbito. Isto implica em dificuldades maiores e custos mais elevados Kahn et al., (1988).

Jencks e colaboradores (1988) listam os seguintes argumentos na defesa do período de internação hospitalar como o momento ideal para medir os óbitos ocorridos, que justificam a coleta exclusiva dos óbitos intra-hospitalares: 1) a diferença no tempo de permanência pode refletir, parcialmente, as diferenças nas necessidades médicas dos pacientes; 2) permanências hospitalares mais prolongadas podem ser resultado de cuidado inefetivo e de uma maior incidência de complicações intrahospitalares; 3 ) a menos que o período de observação seja bastante prolongado, um tempo fixo poderá não contabilizar mortes de pacientes de longa permanência que ainda estão internados; 4) usar um período fixo de observação pode parecer injusto, pois administradores hospitalares, e mesmo médicos, freqüentemente conhecem pouco e têm controle limitado sobre o que acontece com seus pacientes após a alta; 5) medir taxas de mortalidade ape- nas durante o período de internação pode ser mais factível, mais fácil e menos oneroso.

Contudo, a controvérsia quanto ao período ideal para medirem-se as taxas de mortalidade hospitalar ainda não foi resolvida. Vários trabalhos, usando as duas abordagens, não encontraram diferenças significativas. O Stanford Center for Healthcare Research (SCHCR, 1976) promoveu um estudo para avaliar se existiam diferenças significativas entre períodos de tempo para o cálculo do número de óbitos e obteve resultados similares entre as taxas de óbito medidas durante o período de internação e as que contabilizaram o total de óbitos 40 dias após a admissão (mesmo após a alta hospitalar). Da mesma forma, DesHarnais et al., (1988), analisando os dados do relatório da HCFA, de 1986, encontrou alta correlação entre as taxas de mortalidade intrahospitalar e as correspondentes aos 30 dias após a admissão, concluindo que ambas as medidas podiam ser utilizadas. Já Chassin e colaboradores (1989) trabalharam com quatro diferentes períodos no cálculo de taxas de mortalidade hospitalar para verificar a probabilidade de morrer em condições médicas específicas: 1) a morte ocorrida durante o período de internação; 2) a morte ocorrida até 30 dias após a alta; 3 ) a morte até 30 dias após a admissão, ocorrida dentro ou fora do hospital; 4) mortes ocorridas até o percentil 95 do tempo médio de permanência para cada condição médica escolhida. As diferenças entre os hospitais com as diferentes medidas utilizadas foram grandes, e significativas estatisticamente, tanto em condições específicas como para o conjunto destas.

A discussão em torno do nível de agregação dos dados aponta para duas estratégias no cálculo das taxas de mortalidade: 1) aquela cujo nível de agregação é o hospital - incluindo todas as internações ocorridas no hospital em um determinado período de tempo, e 2) aquela onde o nível de agregação é um diagnóstico ou procedimento específico - incluindo as internações referentes a procedimentos médicos ou categorias específicas de diagnósticos, ocorridas no hospital em um determinado período de tempo.

As análises que usam o hospital como universo de agregação têm sido capazes de explicar melhor a variação da mortalidade entre serviços do que as que trabalham com uma enfermidade específica. As análises de hospitais explicaram de 35 a 93\% da variação observa- 
da entre serviços. Este resultado se deve ao fato de que se observa menos variação aleatória usando dados de um hospital (OTA, 1988). Entretanto, em estudos mais recentes, prevalece a opção pela desagregação dos dados de procedimentos ou diagnósticos para melhorar a validade do indicador.

Um outro aspecto a ser considerado é o fato de ser a morte um evento relativamente raro. Assim, mesmo quando se analisam as taxas de mortalidade em diagnósticos que apresentam letalidade relativamente alta, o número de pacientes que morrem durante ou imediatamente após a hospitalização pode ser muito pequeno. Isto é particularmente válido para as faixas etárias mais jovens. Para reduzir esta limitação, vários estudos agregam internações de muitos anos para o cálculo de suas taxas de mortalidade.

A qualidade dos dados é fundamental para garantir a validade do indicador. Duas fontes de dados principais são utilizadas nos estudos sobre mortalidade hospitalar: as bases de dados administrativos e os prontuários médicos. As análises da mortalidade hospitalar que se utilizam de bases de dados administrativos partem do pressuposto de que os hospitais utilizam procedimentos padronizados de coleta, codificação, definição de terminologia, classificação e nomenclatura para produzir essas informações. Mas raramente isto realmente acontece. Pode ocorrer grande variação na qualidade dos dados entre hospitais, afetando substancialmente os estudos comparativos (OTA, 1988). A produção de dados confiáveis exige padronização e atualização, o que implica em esforço apropriado e permanente para a melhoria da qualidade dos dados.

A coleta de dados a partir dos prontuários médicos pode ser necessária para a obtenção de dados clínicos dos pacientes, mas existem problemas associados a este processo. A quantidade e a qualidade de informações disponíveis nos prontuários médicos podem variar entre profissionais, hospitais e períodos de tempo. Portanto é necessário estabelecer critérios de coleta de dados mais precisos para contornar as diferenças entre a linguagem médica e as práticas de registro.

Algumas das vantagens do uso das bases de dados administrativos são a cobertura alta, o custo relativamente baixo e a flexibilidade de uso em diversos desenhos de pesquisa. As desvantagens são a freqüente falta de disponibilidade de dados clínicos e a precária confiabilidade das informações, principalmente da informação diagnóstica (Flood, 1990; Lohr, 1990; DesHarnais, 1990a; Travassos \& Martins, 1994). Embora as bases de dados de serviços de saúde possam, no futuro, incorporar uma gama bem maior de variáveis clínicas Hornbrook et al., (1998), atualmente elas nem sempre contêm informações detalhadas, que podem ser necessárias para o ajuste das taxas de mortalidade, como veremos a seguir.

Chassin et al., (1989a) salientam que um hospital com uma boa qualidade pode, em tese, documentar melhor suas internações que hospitais que produzem atenção de padrão inferior. Deste modo, a anotação do diagnóstico secundário é outro tópico bastante discutido, principalmente no que diz respeito à integralidade das anotações nos prontuários médicos e nas bases de dados. Por exemplo, quando o diagnóstico secundário é usado como variável de ajuste do risco de morrer, a falha na documentação dessa informação afeta diretamente o cálculo da mortalidade esperada. Sobretudo nos Estados Unidos, as preocupações com a melhoria desse tipo de informação levaram à ampliação do número de campos destinados ao registro dos diagnósticos secundários e ao posterior aumento do uso desses campos. Em 1989, já havia um percentual bastante significativo de casos com cinco diagnósticos registrados, número bastante superior ao obtido em 1987 (Iezzoni, 1994).

\section{A gravidade do caso}

Nas análises comparadas do desempenho hospitalar, a influência da gravidade dos casos é destacada como importante fator de confusão (Hornbrook \& Monheit, 1985; Horn et al., 1985; Aronow, 1988; Green et al., 1990; Iezzoni, 1994). A mensuração precisa da gravidade dos casos requer, na maioria das vezes, a incorporação de diversas variáveis clínicas nem sempre disponíveis nos bancos de dados secundários, como citado anteriormente, o que leva os pesquisadores a utilizarem estratégias diferentes.

Apesar de a gravidade da doença ser a variável que melhor explica a utilização de recursos hospitalares, o conceito de gravidade expressa mais uma dimensão de risco do que de consumo de recursos. Na mensuração da gravidade são adotadas abordagens diferentes, de acordo com os objetivos do estudo e 
com o autor. Algumas definições estão relacionadas à probabilidade de morte, outras à perda total ou parcial de função orgânica, ou ainda à intensidade da doença (burden of illness) e à integridade fisiológica (Spiegel \& Kavaller, 1986; Thomas, 1990).

Diferenças no perfil de gravidade dos casos entre hospitais podem ocorrer devido a: 1) complexidade tecnológica; 2) hospitais que preferencialmente admitem pacientes menos graves; 3 ) diferenças nos critérios de hospitalização; e 4) características da oferta de serviços.

A gravidade pode diferir consideravelmente entre categorias diagnósticas e grupamento de diagnósticos. Considera-se a identificação do diagnóstico principal do paciente como uma dimensão essencial para o ajuste de risco. Um outro elemento importante é a diferenciação de pacientes classificados em um mesmo diagnóstico principal que apresentam graus de gravidade distintos, através da verificação da presença de patologias associadas e da verificação da ocorrência de variações na gravidade da própria doença.

Com relação às patologias associadas, (DesHarnais et al., 1988) ressaltam que o peso dos diagnósticos secundários (comorbidades) na gravidade do caso varia segundo o diagnóstico principal, sendo que certas combinações de condições mórbidas apresentam maior risco que outras. Outro aspecto que deve ser destacado é a inclusão de diagnósticos secundários resultantes de iatrogenia (complicações) nas medidas de gravidade, pois desta forma, misturam-se fatores de risco do paciente com aspectos referentes do desempenho hospitalar que se quer avaliar (Greenfield et al., 1988). Nesse sentido, embora muitas vezes seja difícil distinguir uma comorbidade de uma complicação, deve-se sempre tentar a exclusão das complicações mais comuns. Uma estratégia para isto seria medi-las na admissão (Chassin et al.,1989a).

Os fatores de risco de morrer do paciente (gravidade) são variáveis de ajuste das taxas de mortalidade hospitalar na realização de estudos comparativos. Segundo Blumberg (1986), um ajuste adequado é muito importante neste tipo de estudo, pois pacientes com características de saúde diferenciadas, que implicam em diferentes tratamentos, não podem ser analisados como similares. Este autor define ajuste como um procedimento expresso no cálculo de taxas ou medidas de associação, a partir do qual os efeitos das diferenças na composição das variáveis nas populações comparadas são removidas por procedimentos estatísticos.

A seleção de variáveis para o ajuste deve ser baseada inteiramente nas características dos pacientes, excluindo-se as características institucionais. Também é essencial para a comparação dos resultados considerar o ajuste dos atributos dos pacientes, anteriores à assistência, que podem influenciar no resultado. Ou seja, para serem feitas comparações entre serviços e prestadores, é necessário o ajuste pelos fatores de risco de morrer dos pacientes.

A idade tem sido utilizada como uma das variáveis de controle da gravidade do paciente, pois existe uma clara associação entre a idade e o risco de morte (OTA, 1988). Iezzoni (1994) sublinha que a idade pode ter um efeito independente dos outros atributos do paciente no risco de morrer, porém este efeito pode depender das categorias de idade e do tipo de indicador de resultado utilizados no estudo. Pacientes muito idosos -80 anos e mais - são considerados um grupo especial, pois diferem fisiologicamente dos mais jovens, mesmo quando outros fatores, como a gravidade da doença, são comparáveis.

O sexo também é freqüentemente utilizado no ajuste das taxas de mortalidade hospitalar, apesar de ser uma das variáveis com menor influência no risco de morrer. Entretanto, para condições específicas, como cirurgia coronariana ou infarto do miocárdio, o ajuste por sexo é importante, pois, nestes casos, o risco de morrer difere entre homens e mulheres (Tofler et al., 1987; Hannan et al., 1994). Vale lembrar que tanto a idade quanto o sexo são variáveis simples e diretas, além de apresentarem boa validade e alta confiabilidade.

A ocorrência de instabilidade clínica aguda também é importante, sendo crucial nas avaliações que envolvem pacientes graves. A instabilidade clínica reflete alterações no estado fisiológico do paciente, sendo resultado de várias medidas, como sinais vitais, balanço hidro-eletrolítico, hematologia, oxigenação arterial, nível de consciência e exame neurológico. É indiscutível a importância dos parâmetros fisiológicos agudos na avaliação do risco de morrer. Mas a obtenção de dados fisiológicos completos requer, na maioria das vezes, uma revisão detalhada do prontuário médico (Iezzoni, 1994).

No ajuste das taxas de mortalidade hospitalar o tipo de admissão pode ser utilizado co- 
mo uma variável proxy da gravidade das condições clínicas do paciente. De fácil obtenção, pode ser utilizada na ausência de variáveis clínicas detalhadas. Esta variável incorpora informações, como se a internação foi eletiva ou de emergência e, em alguns bancos de dados, se a admissão resulta de transferência de outra instituição. Os pacientes que tiveram uma admissão eletiva, em geral, apresentam melhor prognóstico do que os pacientes que foram admitidos por emergência. Já os pacientes transferidos de hospitais crônicos, via de regra, são casos graves ou terminais e, portanto, têm maior risco de morrer.

A condição social do paciente é uma característica fundamental para a avaliação dos serviços de saúde e pode explicar variações no acesso e na adequação do cuidado (Towsend \& Davidson, 1982; Victoria et al., 1988; Monteiro, 1988; Morris, 1990; Travassos Veras, 1992). No tocante à mortalidade hospitalar, a associação entre a morte no hospital e a classe social do paciente pode ser resultado de desigualdades na qualidade do cuidado prestado a indivíduos socialmente distintos. Assim, sempre que se supõe que a condição social do paciente possa impactar negativamente sobre a qualidade do processo de cuidado, ela não deve ser incorporada como variável de ajuste do risco de morrer, pois anularia o seu efeito sobre as taxas de mortalidade.

A relação entre tempo de permanência do paciente no hospital e mortalidade é mais complexa. Variações no tempo de permanência podem expressar diferenças na gravidade dos casos ou, por outro lado, refletir baixa qualidade do atendimento. A utilização desta variável no ajuste do risco de morrer se justifica pelo fato de apresentar padrões diferenciados entre áreas e prestadores. Entretanto, Blumberg (1987) ressalta que a utilização do tempo médio de permanência como variável independente em modelos preditivos do risco de morte, tende a favorecer os hospitais com maior tempo de permanência, através de uma taxa de mortalidade estimada maior. Os opositores desta posição ressaltam que a qualidade e a permanência estão relacionadas, ou seja, o hospital pode ter um longo tempo médio de permanência por prestar assistência de má qualidade. Neste caso, a permanência não deveria ser usada no ajuste do risco de morrer, como qualquer outro fator que tenha uma potencial inter-relação com a qualidade da assistência Chassin et al., (1989a). Como será discutido adiante, alguns autores ressaltam que o tempo de permanência mostra-se inválido como variável de ajuste para hospitais, mesmo quando corrigido pela taxa de ocupação como foi utilizado por Roemer et al. (1968) e criticado por Goss e Reed (1974). Outros autores observam a baixa correlação entre mortalidade hospitalar e tempo de permanência (Ducket \& Kristoffereson, 1978; Dubois, 1987a).

Por fim, o ajuste das taxas pode considerar a inclusão do estado funcional do paciente. Alterações no estado funcional podem ter diversas expressões, tais como, limitações sensoriais e motoras, físicas e psicológicas. O estado funcional está associado a várias medidas de resultado dentre elas a morte eminente. Keeler e colaboradores (1990) encontraram que a incapacidade de deambular antes da admissão é um preditor de óbito, até 30 dias após admissão, para pacientes com pneumonia. Alterações no estado funcional encontram-se também associadas ao maior risco de complicações pós-operatórias e maior risco de morrer para condições específicas como o câncer de pulmão e o acidente cerebrovascular (Iezzoni, 1994).

\section{Classificações de gravidade}

Embora, atualmente, poucos discordem da necessidade de se ajustarem as taxas de mortalidade para graus diferenciados de gravidade (Horn, 1983; Shachtman, 1986; Dubois, 1987b; Flood \& Scott, 1987; OTA, 1988; Hartz et al., 1989; Thomas \& Ashcraf, 1989; Fink et al., 1989; Luft et al., 1990; Burns \& Wholey, 1991; Kahn, 1992; Keeler et al., 1992; Siber, 1992; Shapiro, 1994; Iezzoni, 1994), não há uma maneira efetiva de fazê-lo.

Existem vários instrumentos ou sistemas de classificação em uso para detectar e mensurar variações na gravidade dos casos e refinar os ajustes nos tipos de casos tratados nos hospitais, permitindo comparações de taxas de mortalidade entre hospitais. Os sistemas mais refinados de classificação de gravidade requerem a incorporação de variáveis clínicas, nem sempre disponíveis nos bancos de dados secundários, o que representa uma dificuldade para o seu uso. Alguns sistemas têm sido desenvolvidos para aplicação em bancos de dados administrativos como, por exemplo, o Índice de Comorbidade de Charlson (Charlson, 1987; Martins \& Travassos, 1998) 
Diversos sistemas de classificação foram construídos para medir a gravidade do paciente. Estes sistemas variam em função das características e dos métodos empregados para classificar os pacientes, da definição de gravidade e dos dados necessários para o seu emprego. Nesses sistemas incluem-se os seguintes: $P a$ tient Severity of Ilness Index - PSI proposto por Susan Horn derivado do AS-SCORE; Disease Staging - DS proposto por Gonnela; Patient Magement Categories - PMC proposto por Young; Medical Ilness Severity Grouping System - MEDISGRPS proposto por Brewster e Jacobs; e Relative Intensity Measures - RIMs proposto por Caterinichio. Entre os vários sistemas dedicados a esta finalidade, os mais utilizados são: o DS, o PMC, o PSI, o sistema APACHE (Acute Physiologic And Chronic Health Evaluation) e o sistema MEDISGROUP (Aronow, 1988; Iezzoni, 1994).

Como apontado anteriormente, não existe ainda consenso sobre o melhor sistema de avaliação da gravidade dos casos. Iezzoni e colaboradores (1996) examinaram em trabalho recente como as taxas de mortalidade intrahospitalar são afetadas por diferentes métodos de ajuste de gravidade. Eles verificaram que as catorze metodologias testadas concordavam sobre o desempenho relativo dos hospitais mais freqüentemente do que seria esperado pelo acaso. Contudo, os diferentes métodos de classificação produziam avaliações bastante diferentes do desempenho entre os hospitais, indicando a necessidade de aprimoramento nas metodologias voltadas para esta finalidade.

\section{Os modelos para o ajuste das taxas de mortalidade}

Os estudos que utilizam a taxa de mortalidade como indicador de qualidade recorrem a diversas estratégias de padronização, visando a compensar a variação na gravidade dos casos analisados (risco de morrer associado ao paciente). Diversos modelos vêm sendo testados para ajustar as taxas de mortalidade pelo tipo de paciente, baseados em dados secundários, informatizados ou não (Roemer et al. 1968; Goss \& Reed, 1974; Ducket \& Kristofferson, 1978; Hebel et al., 1982; Blumberg 1986, 1988; Dubois et al., 1987a, b; DesHarnais et al., 1988, 1990; HCFA, 1988; Chassin et al., 1989a; Hartz et al., 1989; Kahn et al., 1990b; Iezzoni 1997;
Wray et al., 1997). Esses modelos buscam dar conta das características demográficas e clínicas do paciente, e fazer correções de diferenças na gravidade da doença principal ou das patologias associadas. A introdução gradual de variáveis relacionadas às características da demanda atendida representa um importante passo na evolução desses modelos.

Em geral, esses modelos são utilizados para a obtenção das probabilidades de óbito a partir da qual se calculam as taxas ajustadas de mortalidade esperada para cada hospital ou prestador. Os prestadores são classificados segundo o seu desempenho com base na relação entre as taxas esperadas e as taxas observadas.

Para a presente revisão foram selecionados três estudos com o objetivo de apresentar a evolução metodológica dos trabalhos sobre a mortalidade hospitalar como indicador de qualidade. Estes estudos foram realizados por Roemer et al., (1968), Ducket e Kristofferson (1978) e Dubois (1987a).

Roemer et al. (1968) utilizaram para ajustar as taxas de mortalidade medidas indiretamente relacionadas à gravidade, ao tempo médio de permanência e à taxa de ocupação. Assim, eles são os primeiros autores a reconhecer a relevância dos ajustes pela gravidade dos casos tratados, de forma que a mortalidade reflita a qualidade da assistência prestada e busque formas de ajuste na análise das variações entre hospitais.

Ducket e Kristofferson (1978) estudando uma amostra de 33 hospitais de Los Angeles, EUA - excluídos os casos obstétricos e perinatais - demonstraram que o tempo de permanência, corrigido pela taxa de ocupação, explicava cerca de $79 \%$ da variação da mortalidade hospitalar entre os hospitais.

Goss e Reed (1974) questionam este resultado e ressaltam que o ajuste utilizado não levou em conta as diferenças regionais, as características da população e os padrões de disponibilidade e de utilização dos hospitais. Ponderam ainda que a taxa de mortalidade para hospitais gerais de curta permanência em diferentes regiões não depende somente da qualidade do cuidado mas, também, da distribuição etária da população e da disponibilidade e do uso de serviços de saúde alternativos. Apontam que o uso da taxa de ocupação corrigida pelo tempo de permanência como medida da gravidade dos casos é questionável. Considerando esta abordagem simplista, estes autores sugerem o uso de taxas de mortalidade desagre- 
gadas por diagnóstico, como uma medida mais específica, que pode ser ajustada estatisticamente pela idade, sexo e outros fatores relevantes.

Ducket e Kristofferson (1978) tentam avançar com base nas conclusões e observações de Roemer et al., (1968) e Goss e Reed (1974). Partem do princípio que o tempo médio de permanência, por ser uma medida de processo, não reflete somente a condição do paciente mas, também, a eficiência do hospital. Em uma amostra de 33 hospitais australianos encontraram uma fraca associação entre o tempo médio de permanência e a mortalidade $(R=0.15)$. Eles propõem uma reformulação ao modelo de Roemer e colaboradores, mas restringem-se à incorporação de variáveis de morbidade e idade como variáveis de ajuste. Os autores denominaram sua proposta de Death Rate Adjusted for Severity of Admissions and Age.

Tanto Roemer et al., (1968) como Ducket e Kristofferson (1978) utilizaram a técnica de padronização indireta para o cálculo das taxas de mortalidade. A segunda proposta, porém, apresenta avanços ao incorporar variáveis diagnósticas e demográficas no ajuste das taxas. Essa evolução tem como pano de fundo a discussão travada na época, segundo a qual o diagnóstico passa a ser entendido como uma informação crucial na explicação das variações das taxas entre serviços ou prestadores.

O terceiro modelo foi desenvolvido por Dubois et al., (1987a) numa conjuntura posterior, o que facilitou a incorporação de variáveis mais refinadas ao modelo de ajuste. Este estudo trabalha com resumos de alta hospitalar e coleta informações sobre as características demográficas e diagnósticas dos pacientes, o tipo de admissão, a origem do paciente e as características do hospital. As variáveis foram: idade (nas seguintes faixas etárias: $<14,14-44$, 45-64, 65-69, >70 anos); o índice do case mix hospitalar calculado com base na distribuição dos casos por Diagnosis Related Groups (DRG); o percentual de admissões de emergência; as readmissões no mesmo hospital em até catorze dias, as admissões provenientes de hospitais de pacientes crônicos; o tamanho do hospital - número de leitos; taxa de ocupação; tempo médio de permanência; e número de admissões em seis meses - o percentual de procedimentos clínicos, as altas para hospitais de pacientes crônicos e as transferência para hospitais de pacientes agudos.
Essas variáveis foram utilizadas para ajustar a taxa de mortalidade de cada hospital, visando a identificar os hospitais cuja diferença entre a taxa observada e a esperada era significativa. A taxa de mortalidade somente apresentou correlação com a distribuição etária (proporção de pacientes com mais de 70 anos, $\mathrm{R}=0.75$ ); o caráter da internação (admissões de emergência, $\mathrm{R}=0.53$ ); admissões de hospitais crônicos $(\mathrm{R}=0.29)$; e o case-mix $(\mathrm{R}=0.55)$.

A regressão múltipla foi usada para prever a mortalidade em cada hospital em relação ao percentual de pacientes com idade superior a 70 anos, ao percentual de admissões de emergência, de admissões de hospitais crônicos (nursing home) e ao índice de case mix. Esse modelo explicou $64 \%$ da variação na mortalidade hospitalar entre serviços. Segundo Dubois (1987a), a inclusão do tempo médio de permanência, do tamanho do hospital, da taxa de ocupação e da transferência para hospitais de crônicos não serviram para aperfeiçoar o modelo. A mortalidade observada excedeu a prevista em onze hospitais e foi muito abaixo do esperado em nove hospitais, considerados desviantes (dez categorias diagnósticas - DRGs); 92\% dos casos estavam relacionados a pacientes beneficiários do programa Medicare e, em cada categoria diagnóstica, a mortalidade hospitalar dos high outliers excederam a dos low outliers.

As diferenças metodológicas entre as três propostas expressam o aprimoramento gradativo dos modelos. Eles avançam substantivamente na forma de abordar a gravidade. Mas ainda permanecem simples em relação à utilização de dados rotineiros ou facilmente disponíveis e operacionalizáveis. A busca da "simplicidade" foi uma tônica que marcou essas propostas e cuja importância pode ser dimensionada na reflexão de Dubois et al., (1987a). Este autor ressalta que diante da importância de monitorar a qualidade da assistência prestada - e considerando a inviabilidade de se avaliarem todos os hospitais ao mesmo tempo, um método de screening, com base no uso de taxas de mortalidade hospitalar, usando dados rotineiros, pode racionalizar o processo através da seleção de um conjunto menor de hospitais a serem objeto de revisão detalhada de seu desempenho. 


\section{Modelos explicativos da variação na mortalidade}

Segundo Fink e colaboradores (1989), até a primeira metade da década de 80 , um número importante de trabalhos publicados apresentava limitações metodológicas e raros estudos buscaram estratégias para aumentar a validade da mortalidade hospitalar como indicador de qualidade (Thomas, 1990; Romano, 1993; Iezzoni, 1995), sendo os três estudos citados anteriormente considerados exceções. Essa produção aumenta expressivamente a partir da segunda metade da década de 80 . Os estudos ganham maior rigor metodológico, principalmente em função da criação de sistemas de classificação de gravidade do caso. $\mathrm{O}$ desenvolvimento de grande bancos de dados informatizados incentiva a pesquisa na área de classificação de gravidade, com impacto positivo sobre os estudos de mortalidade hospitalar.

A comparação entre os estudos relacionados com a mortalidade hospitalar é difícil, pois utilizam um conjunto bastante diversificado de variáveis para controlar a gravidade dos casos e técnicas estatísticas diferentes. As variáveis mais freqüentemente utilizadas para ajustar o perfil de casos tratados são: idade, raça, sexo, fonte de financiamento, tipo de seguro saúde, realização de procedimento cirúrgico e tipo de admissão (Blumberg, 1987; Greenfield et al., 1988; OTA, 1988; Luft et al., 1990). Por outro lado, estudos que utilizam classificações de gravidade dos casos no ajuste das taxas de mortalidade indicam que os resultados obtidos são sensíveis à classificação empregada, isto é, o desempenho dos hospitais variam segundo o tipo de classificação utilizada (Iezzoni, 1994; Iezzoni et al., 1995, 1996; Shapiro, 1994). Dessa forma eles reforçam a necessidade de aprofundamento da pesquisa na área de classificação de gravidade.

Fink e colaboradores (1989) descrevem quatro linhas básicas de estudos sobre os determinantes da variações na qualidade do cuidado medidas através da mortalidade hospitalar: 1) estudos que examinam como o tamanho do hospital e o volume dos serviços produzidos afetam a qualidade da assistência; 2) estudos que examinam como as características dos médicos e dos hospitais explicam as variações nas taxas de mortalidade hospitalar; 3) estudos que examinam como as características dos pacientes explicam as variações nas taxas de mortalidade; e 4) estudos que comparam o desempenho entre hospitais universitários e hospitais não universitários.

Várias pesquisas apontam que a mortalidade hospitalar varia acentuadamente entre hospitais, mas produzem resultados contraditórios sobre as causas dessa variação. Estudos que buscam identificar os determinantes dos problemas na qualidade do cuidado nos hospitais apontam diversas características associadas à qualidade, quando medida através de taxas de mortalidade (SCHCR, 1976; Kelly \& Hellinger, 1986; OTA, 1988; Fink et al., 1989; Hartz et al., 1989; Luft et al., 1990; Martins 1991; Burns \& Wholey, 1991; Keeler et al., 1992; Noronha, 1996).

Alguns destes estudos demonstram que o volume de procedimentos realizados no hospital é negativamente associado com a taxa de mortalidade ou, dito de outra forma, que o volume é positivamente associado com a qualidade do cuidado (Flood et al., 1984 a,b; Flood \& Scott, 1987; Roos et al., 1987; Showstack et al., 1987; Wennberg et al., 1987; Kelly 1990; Luft et al., 1990; Noronha, 1996). Outros estudos analisaram a associação entre a qualidade do cuidado e a formação e o treinamento profissional. O grau de organização e comunicação do corpo clínico, a experiência e a especialização dos profissionais, aparecem associados à melhoria de qualidade (Lee et al., 1957; Ashley et al., 1971; Horn 1983; Horn et al., 1985; Kelly \& Hellinger, 1986; Shortell 1988; OTA 1988; Hartz et al., 1989; Kuhn et al., 1994). Com relação à natureza jurídica observou-se um padrão de cuidado melhor nos hospitais não lucrativos (Burns et al., 1993; Shapiro et al., 1993; Kuhn et al., 1994). Os hospitais com maior estabilidade financeira também tiveram um desempenho melhor (OTA, 1988).

\section{Comentários finais}

É necessário destacar a importância do desenvolvimento de estratégias para permitir que os resultados das avaliações melhorem a efetividade dos cuidados hospitalares. A finalidade do uso de indicadores de desempenho consiste em identificar e analisar problemas no cuidado e na promoção de ações de saúde, buscando a modificação e a reversão da situação encontrada. O cuidado à saúde pode melhorar se as avaliações forem utilizadas como informação educativa para ações de aperfeiçoamento. 
Nenhuma característica do cuidado em saúde está mais estreitamente ligada à missão das instituições de saúde que as intervenções para evitar ou retardar a morte. Desta maneira, embora o emprego da taxa de mortalidade hospitalar como indicador da qualidade do cuidado possa apresentar problemas em sua validade, este indicador representa uma informação que os hospitais, os profissionais e os financiadores devem usar para melhor entender o processo de atendimento ao paciente, orientando seu aprimoramento.

Para que estes dados estimulem o aprimoramento da qualidade, torna-se necessário romper barreiras importante nas atitudes e reações dos administradores hospitalares e médicos, e demais profissionais de saúde, clientes potenciais dessa informação. Neste sentido, é bastante ilustrativo o estudo realizado por Berwick e Wald (1990) sobre as opiniões e atitudes dos administradores hospitalares em relação à publicação dos dados de mortalidade pelo HCFA. Todos os hospitais, independentemente de suas taxas de mortalidade, compartilhavam uma visão extremamente negativa sobre o rigor, a utilidade para os consumidores, e sobre a capacidade de interpretação dos dados de mortalidade hospitalar publicados por aquele órgão. Mas apesar de um percentual razoável de instituições citar problemas causados pela publicação dos dados, elas também relataram tê-los utilizado para guiar seus esforços na melhoria da qualidade.
Algumas ações por parte das autoridades de saúde podem ajudar a aperfeiçoar a receptividade dessas informações e a difusão de ações para a utilização de medidas para melhorar a assistência. Entre elas, poder-se-iam arrolar: 1) esforços contínuos para a melhoria da qualidade da própria informação fornecida, de maneira que as taxas espelhem melhor as diferenças reais na qualidade do cuidado, reduzindo a ocorrência de classificações equivocadas das instituições hospitalares; 2) suporte técnico aos hospitais para coletarem, analisarem e interpretarem seus indicadores de desempenho como meio para o aprimoramento da qualidade; 3 ) suporte técnico para desenvolvimento de bancos de dados que permitam a realização de estudos comparativos do desempenho entre hospitais e prestadores; 4) facilitação de interações entre as instituições hospitalares com o propósito de trocar experiências sobre atividades de melhoria do cuidado.

Concluindo, embora ainda existam questões conceituais e metodológicas a serem aprimoradas, as taxas de mortalidade hospitalar devem ser consideradas uma ferramenta útil e factível para discriminar serviços e prestadores com desempenho diferenciado. Sua utilização permite uma primeira detecção de problemas com a qualidade dos cuidados prestados, possibilitando que instituições sejam identificadas e processos de melhoria sejam instituídos para que questões de qualidade responsáveis pela ocorrência de óbitos passíveis de prevenção, sejam sanadas.

\section{Referências}

Almeida C 1997. Os modelos de reforma sanitária dos anos 80: uma análise critica. Saúde e Sociedade 5(2): 3-57.

Anderson GF, Erickson JE 1987. National medical care spending. Health Affairs Fall: 96-104.

Aronow DB 1988. Severity-of-ilness measurement: applications in quality assurance and utilization review. Medical Care Review 45(2): 339-366.

Ashey JSA et al. 1971. Case fatality of hyperplasia of the prostate in two teaching and three regional board hospitals. Lancet Dec: 1308-1311.

Berwick D 1996. Primer on leading improvements of systems. Bristish Medical Journal 312: 619.

Beriwick DM, Wald DL 1990. Hospital leaders opinions of the HCFA mortality data. JAMA 263(2): 247-249.

Blumberg MS 1986. Risk adjusted health care outcomes: a methodologic review. Medical Care Review 43(2): 351-393. 
Blumberg MS 1987. Comments on HCFA hospital death rate statistical outliers. Health Services Research 21(6): 715-739.

Blumberg MS 1988. Measuring surgical quality in Maryland: a model. Health Affairs 25: 62-78.

Brook RH et al. 1977. Assessing the quality of medical care using outcome measures: an overview of the methods. Medical Care (Suppl.) 15(9): 1-106.

Brook RH, McGlynn EA 1991. Maintaining quality of care. In E Ginnzberg, Health Services Research Key to Health Policy. Haward University Press, Cambridge, MA.

Burns LR, Wholey D 1991. The effects of patient, hospital, and physician characteristics on lenght of stay and mortality. Medical Care 29(3): 251-271.

Burns LR et al. 1993. Mortality in a public and a private hospital compared: the severity of antecedent disorders in Medicare patients. American Journal of Public Health 83(7): 966-971.

Charlson ME, Pompei P, Aleski KL, Makenzie R 1987. A new method of classifying prognostic comorbidity in longitudinal studies: development and validation. Journal of Chronic Disease 40: 373-383.

Chassin M et al. 1989a. Differences among hospitals in Medicare patient mortality. Health Service Research 24(1): 1-31.

Chassin $\mathrm{M}$ et al. 1989b.Characteristics of clinical indicators. QRB November: 330-339.

Cleves MA, Golden WE 1996. Assessment of HCFA's 1992. Medicare hospital information report of mortality following admission for hip arthroplasty. Health Service Research 31(1): 39-48.

Codman E 1916. Hospital standartization Surg Gynecol Obstet, 1916, apud ST Normand, ME Glickman, RG Sharma \&BJ McNeil 1996. Using admission characteristics to predict short-term mortality from myocardial infarction in elderly patients. Results from the Cooperative Cardiovascular Project. JAMA 275(17): 1322-1328.

Contandriopoulos AP 1994. Réformer le système de santé: une utopie pour sortir d'un statu quo impossible. Ruptures 1(1): 8-26.

DesHarnais S et al. 1988. The risk-adjusted mortality index a new measure of hospital performance. Medical Care 26(12): 1129-1148.

DesHarnais S et al. 1990a. Measuring hospital performance. Medical Care 28(12): 1127-1141.

DesHarnais S 1990b. Current uses of large data sets to assess the quality of providers. International Journal of Tecchonoly Assessment in Health Care 1(6): 229-238.

Dillner L 1995. Scottish death rates published with health warning. British Medical Journal 309: 1599-1600, apud J Mant \& N Hicks 1994. Detecting differences in quality of care: the sensitivity of measures of processes and outcomes in treating acute myocardial infarction. British Medical Journal 311(7008): 793-796.

Donabedian A 1980. The Definition of Quality and Approaches to its Assessment. Health Administration Press, Ann Arbor, MI.

Donabedian A 1985. The Methods and Findings of Quality Assessment and Monitoring: an Ilustrated Analysis. Health Administration Press, Ann Arbor, MI.

Dubois RW, Brook RH 1988. Preventable deaths: who, how often, and why? Ann Intern Med 109(7):582589.
Dubois R et al. 1987a. Adjusted hospital death rates: a potential screen for quality of medical care. American Journal of Public Health 77(9): 1162-1166.

Dubois R et al. 1987b. Hospital inpatient mortality - Is it a predictor of quality? New England Journal of Medicine317(26): 1674-1680.

Ducket SJ, Kristofferson SM 1978. An index of hospital performance. Medical Care 16(5): 400-407.

Editorial 1993. Lancet 341: 1183-1184.

Fink A et al. 1989. The condition of the literature on differences in hospital mortality. Medical Care 27(4): 315-336.

Flood AB 1990. Peaks and pits of using large data bases to measure quality of care. International Journal of Techonology Assessment in Health Car 6: 253-262.

Flood AB, Scott WR 1987. Hospital Structure and Performance. The Johns Hopkins University Press, Baltimore.

Flood AB et al. 1984a. Does practice make perfect? Part 1 : the relation between volume and outcome for selected diagnostics. Medical Care 22(2): 98-114.

Flood AB et al. 1984b. Does practice make perfect? Part 2: the relation between volume and outcome and other hospital characteristics. Medical Care 22(2): 115-125.

Fottler MD 1987. Health care organization performance: present and future research. Journal of Management 13(2): 367-391.

Fottler MD, Slovensky DJ \& Rogers SJ 1987. Public release of hospital specific death rates - Guidelines for health care executives. Hospital \& Health Services Administration August: 343-357.

Goldfarb MG et al. 1984. Determinats of hospital use: a cross-diagnostic analyses. Medical Care 22:967974.

Goss MEW, Reed JI 1974. Evaluation the quality of hospital through severity-adjusted death rates: some pitfalls. Medical Care 12(3): 202-213.

Green J et al. 1990. The importance of severity of ilness in assessing hospital mortality. JAMA 263(2):241246.

Greenfield S et al. 1988. Flaws in mortality data. The hazards of ignoring comorbid disease. JAMA 260(15): 2253-2255.

Hannan RG et al. 1994. Improving the outcomes of coronary artery bypass surgery in New York State. JAMA 271: 761-766.

Hartz A et al. 1989. Hospital characteristics and mortality rates. New England Journal of Medicine 321(25): 1720-1725.

HCFA - Health Care Financing Administration 1988 U.S. Department of Health and Human Services. Medicare Hospital Mortality Information -87 . HCFA pub. no. 00651, p 1-20.

Hebel JR et al. 1982 Assessment of hospital performance by use of death rates. JAMA 248(23): 3131-3135.

Horn SD 1983. Measuring severity of illness: comparasion across institutions. American Journal of Public Health 73(1): 25-31.

Horn SD et al. 1985. Interhospital diffrences in severity of illness - Problems for prospective payment based on diagnosis related groups. New England Journal of Medicine 313: 20-24.

Horn SD et al. 1991. The relationship between severity of illness and hospital lenght of stay and mortality. Medical Care 29(4): 305-317. 
Hornbrook M, Monheit AC 1985. The contribution of case-mix severity to the hospital cost-output relation. Inquiry 22: 259-271.

Hornbrook M et al. 1998. Health-based payment and computerized patient record systems. Effective Clinical Practice 1: 66-72.

Hudges RG et al. 1988. Hospital volume and patient outcome: the case of hip fracture patients. Medical Care 26(11): 1057-1067.

Hurst JW 1991.Reforming health care in seven European nations. Health Affairs Fall: 7-21.

Iezzoni L 1994. Risk Adjustment for Measuring Health Care Outcomes. Health Administration Press, Ann Arbor, MI.

Iezzoni L 1997. The risks of risk adjustment. JAMA 278(19): 1600-1607.

Iezzoni L et al. 1995. Using severity-adjusted stroke mortality rates to judge hospitals. International Journal for Quality in Health Care 7(2): 81-94.

Iezzoni L et al. 1996. Severity measurement methods and judging hospital death rates for pneumonia. Medical Care 34(1): 11-28.

Jenks SF et al. 1988. Assessing hospital associated deaths from discharge data: the role of lenght of stay and comorbidity. JAMA 260(5): 2240-2246.

Kahn KL et al. 1988. Interpreting hospital mortality data: how can we proceed? JAMA 260(24): 3625-3628.

Kahn KL et al. 1990a. The effect of the drg-based prospective payment system on quality of care for hospitalized medicare patient - An introduction to the series. JAMA 264(15): 1953-55.

Kahn KL et al. 1990b. Measuring quality of care with explicit process criteria before and after implementation of the DRG-based prospective payment system. JAMA 264(15):1969-1973.

Kahn KL et al. 1990c. Comparing outcomes of care before and after implementation of the DG-based prospective payment system. JAMA 264(15): 19841988.

Keeler EB et al. 1990. Changes in sickness at admission following the introduction of the prospective payment system. JAMA 264(15): 1962-1968.

Keeler EB et al. 1992. Hospital characteristics and quality of care. JAMA 268(13): 1709-1714.

Kelly JV 1990. What do mortality studies reveal about hospital volume, teaching status, and ownership? International Journal of Technology Assessment in Health Care 6: 239-252.

Kelly JV, Hellinger FJ 1986. Physician and hospital factors associated with mortality of surgical patients. Medical Care 24(9): 785-800.

Kind P 1995. Hospital deaths: the missing link. Discussion paper 44. York: Centre for Health Economics, apud J Mant \& N Hicks 1988, detecting differences in quality of care: the sensitivity of measures of processes and outcomes in treating acute myocardial infarction. British Medical Journal 311(7008): 793-796.

Krakaver H et al. 1992. Evaluation of the HCFA model for the analysis of mortality following hospitalization. Health Service Research 27(3):317-335.

Kuhn EM et al. 1991. The relationship of hospital characteristics and the results of peer review in six large states. Medical Care 21(10): 1028-1038.

Kuhn EM et al. 1994. The relationship of hospital ownership and teaching status to 30 and 80 day adjusted mortality rates. Medical Care 32(11): 1098-1108.
Lee JAH, Morrison SL, Morris JN 1957. Fatality from three common surgical conditions in teaching and non teaching hospitals. Lancet (October): 785-790.

Localio AR et al. 1997. The public release of hospital and physician mortality data in Pennsylvania. A case study. Medical Care 35(3):272-286.

Lohr KN 1990. Use of insurance claims data in measuring quality of care. International Journal of Technology Assessment in Health Care 6: 263-271.

Luft HS1980. The relation between surgical volume and mortality: an exploration of causal factors and alternatives models. Medical Care 18(9): 940.

Luft HS, Hunt SS 1986. Evaluating individual hospital quality through outcome statistics. JAMA 255(20): 2780-2784.

Luft HS et al. 1990. Hospital Volume, Physician Volume, and Patient Outcomes: Assessing the Evidence. Health Administration Press Perspectives, Ann Arbor, MI.

Martins MS 1991. Mortalidade Hospitalar: Novas Aborgagens Metodológicas. Dissertação de Mestrado. Escola Nacional de Saúde Pública, Fundação Oswaldo Cruz.

Martins MS, Travassos C 1998. Assessing the availability of case mix information in hospital database systems in Rio de Janeiro, Brazil. International Journal for Quality in Health Care 10(2): 125-133.

McKee M 1997. Indicators of clinical performance. Bristish Medical Journal 312: 619.

Monteiro CA 1988. Saúde e Nutrição das Crianças de São Paulo: Diagnóstico, Contrastes Sociais e Tendências. Hucitec e Universidade de São Paulo, São Paulo.

Morris JN 1990. Inequalities in health: ten years and little further on. Lancet 336: 491-493.

New York State Department of Health 1997. Coronary artery by-pass surgery in New York State, 1993-1995.

Noronha JC 1996. Utilização de taxas de mortalidade hospitalar em hospitais de agudos no Brasil. Memória apresentada à Academia Nacional de Medicina. Rio de Janeiro.

O’Leary Dennis S 1995. Performance measures - How are they developed, validated, and used? Medical Care 33(Suppl.): js13-js17.

OECD - Organization for Economic Cooperation and Development 1995. Directions in health care policy. Health policies studies no. 7. Paris.

OTA - Office of Technology Assessment 1988. Hospital Mortality Rates, p. 71-98. In Quality of Medical Care - Information for Consumers, OTA - H-386. Congress of the United States: Office of Technology Assessment. Washington D.C.

Roemer MI et al. 1968. A proposed hospital quality index: hospital death rates adjusted for case severity. Health Service Research (Summer): 68-118.

Rogers WH et al. 1990. Quality of care before and after implementation of the DRG-based prospective payment system - A summary of effects. JAMA 264(15): 1989-1994.

Romano PS 1993. Can administrative data be used to compare the quality of health care? Medical Care Review 50(4): 451-477.

Ross LL et al. 1987. Monitoring adverse outcomes of surgery using administrative data. Health Care Financing Review (annual suppl.): 5-16.

Roos NP et al. 1988. Using administrative data to predicted important health outcomes. Medical Care 26(3): 221-239. 
Rubenstein LV et al. 1990. Changes in quality of care for five diseases measured by implicit review, 1981 to 1986. JAMA 264(15): 1974-1979.

Saltman RB, Otter C 1989. Public competition versus mixed markets: an analytic comparasion. Health Policy 11: 43-55.

Schieber GJ, Poullier JP 1987. Recent trends in international health care spending. Health Affairs Fall: 105112.

Scott WR 1977. Effectiveness of organizations effectiveness studies, p. 63-95. In PS Goodman \& J M Pennings (eds) New Perspectives on Organizational Effectiveness. Jossey Bass, San Francisco.

Shachtman RH 1986. A method for constructing case mix indexes, with application to hospital length of stay. Health Service Research 20(6): 737-762.

Shapiro MF 1994. The effect of alternative case mix adjustments on mortality differences between municipal and voluntary hospital in New York City. Health Service Research 29(1): 95-112.

Shapiro MF et al. 1993. Mortality differences between New York City municipal and voluntary hospital, for selected conditions. American Journal of Public Health 83(7):1024-1026.

Sheldon T 1998. Promoting health care quality: what role performance indicators? Quality in Health Care 7 (Suppl.): S45-S50.

Shortell SM et al. 1981. Hospital medical staff organization and quality of care: results for myocardial infarction and appendectomy. Medical Care 19(10): 1041-1053.

Showstack JA et al. 1987. Association of volume with outcome of coronary artery bypass graft sugery Scheduled vs. nonscheduled operations. JAMA 257(6):785-789.

Silber JH et al. 1992. Hospital and patient characteristics associated with death after surgery. Medical Care 30(7): 615-629.

Spiegel AD, Kavaller F 1988. Cost Containment and DRGs. A Guide Prospective Payment. Ed National Health Publishing, EUA.

SCHCR - Standford Center for Health Care Research 1976. Comparison of hospitals with regard to out- comes of surgery. Health Services Research (summer): 112-127

Sullivan SW, Hays LB 1989. Meicare Hospital Mortality Information, 1986, 1987, 1988. Health Care Financing Administration, HCFA Publication 00701, US Department of Health and Human Services, Washington, DC.

Thomas JW, Ashcraft MLF 1989. Measuring severity of illness: a comparison of inter-rater reliability among severity methodologies. Inquiry 26: 483-492.

Thomas SW 1990. Application of severity measurement systems for hospital quality management. Hospital \& Health Services Administration 35(2): 221-243.

Tofler et al. 1987. Effect of gender and race on prognosis after myocardial infarction: adverse prognosis for women, particularly black women. Journal of the American College of Cardiology 9: 473.

Travassos Veras CM 1992. Equity in the Use of Private Hospitals contracted by a Compulsory Scheme in the City of Rio de Janeiro, Brazil, in 1986. Ph.D Thesis, London School of Economics and Political Science, London.

Travassos Veras CMT, Martins MS 1994. Confiabilidade dos dados nos formulários de internação hospitalar (AIH), Rio de Janeiro, Brasil. Cadernos de Saúde Pública 10(3): 339-355.

Towsend P, Davidson N 1982. Inequalities in Health: the Black Report. Harmondsworth, London.

Victoria CG, Barros FC, Vaughan JP 1988. Epidemiologia da Desigualdade: um Estudo Longitudinal de 6.000 Crianças Brasileiras. Hucitec, São Paulo.

Wennberg JE et al. 1987. Use of claims data systems to evaluate health care outcomes: mortality and reoperation following prostatectomy. JAMA 257(7):933936.

WHO - World Health Organization 1993. Evaluation of Recent Changes in the Financing of Health Services. WHO Technical Report Series 829, Geneva.

Wray NP et al. 1997. Case-mix adjustment using administrative databases: a paradigm to guide future research. Medical Care Research and Review 54(3): 326-356.

Wyzewianski L 1988. Quality of care: past achievements and future challenges. Inquiry 25: 13-22. 